\title{
BENEFICIOS DE LA ADOPCIÓN DE SEMILLA MEJORADA DE MAÍZ EN LA REGIÓN CENTRAL DE PUEBLA
}

\author{
BENEFITS OF ADOPTION OF IMPROVED MAIZE SEED IN THE PUEBLA CENTRAL REGION
}

\author{
Luis A. Flores-Cruz y J. Alberto García-Salazar*
}

\begin{abstract}
Postgrado en Economía, Campus Montecillo, Colegio de Postgraduados. Km. 36.5 Carr. México-Texcoco. 56230, Montecillo, Texcoco, Estado de México.

*Autor de correspondencia (jsalazar@colpos.mx)
\end{abstract}

\section{RESUMEN}

El conocimiento existente sobre los beneficios que la adopción de semilla mejorada tiene sobre la productividad en maíz (Zea mays L.) es insuficiente, lo que hace necesario realizar más investigación sobre el tema. El objetivo de este proyecto fue medir los beneficios de la adopción de semilla mejorada sobre el bienestar de los productores de maíz en la región central de Puebla integrada por los distritos de Cholula, Libres y Tecamachalco. Se obtuvo la solución de un modelo de equilibrio espacial aplicado al mercado de maíz en 2013, y se estimó un modelo logit agrupado en donde la variable dependiente es el logit de la razón de probabilidades de usar semilla mejorada contra semilla criolla. Los resultados indican que en 2013 la tasa de adopción de semilla mejorada en la región central de Puebla fue de $37.46 \%$. Una disminución en el precio de la semilla mejorada de 5,15 y $25 \%$, manteniendo los demás factores constantes, aumentaría la tasa de adopción de semilla mejorada de maíz en $2.2,6.6$ y $11.1 \%$, respectivamente. Dichos cambios aumentarían el rendimiento de maíz en $0.8,2.5$ y $4.1 \%$, lo que originaría un aumento en el bienestar (medido por el excedente económico total) de los productores y consumidores de maíz por 44, 133 y 222 millones de pesos, respectivamente. Con base en los resultados se recomienda que el gobierno promueva el establecimiento de una estructura competitiva en el mercado de semilla mejorada de maíz.

Palabras clave: Zea mays, adopción, semilla mejorada, beneficio, modelo de equilibrio espacial, modelo logit.

\section{SUMMARY}

Knowledge about the benefits that adoption of improved seed has on productivity in maize (Zea mays L.) is insufficient, necessitating more research on this issue. This project measured the benefit of adoption of improved seed on the welfare of maize growers in the Puebla central region which comprises Cholula, Libres and Tecamachalco districts. The solution of a spatial equilibrium model applied to the maize market was obtained, and a grouped logit model that considers the logit of the probability ratios of using improved seed against using native seed as the dependent variable was estimated. Results indicated that the adoption rate of improved seed in the Puebla Central region was $37.46 \%$. A decrease in the improved seed price by 5 , 15 and $25 \%$, while holding other factors constant, would increase the adoption rate of improved maize seed by $2.2,6.6$ and $11.1 \%$, respectively. Those changes would increase maize yield in $0.8,2.5$ and $4.1 \%$, and the welfare of growers (as measured by economic surplus) would increase by generating 44, 133 and 222 million pesos, respectively. Based on these, it is recommended that the government promotes a competitive structure in the market of improved maize seed.

Index words: Zea mays, adoption, improved seed, benefit, spatial equilibrium model, logit model.

\section{INTRODUCCIÓN}

Actualmente, México presenta el problema de dependencia alimentaria en maíz (Zea mays L.). En el periodo 2013-2015 las importaciones promedio de maíz fueron de 9.23 millones de toneladas, y se espera que en 2025 se ubiquen en 13.8 millones (SIAP, 2016; USDA, 2016). A nivel estatal, Puebla presenta una situación similar. Flores-Cruz et al. (2014) reportaron que durante el periodo 2008-2010 el promedio anual de la producción y el consumo de la entidad fueron 852 y 1,738,000 t, lo que determinó un fuerte déficit por 886,000 t que tuvo que ser abastecido con maíz proveniente de otras entidades y con importaciones.

La dependencia alimentaria de maíz en México y en el estado de Puebla ocasiona vulnerabilidad a las fluctuaciones en los precios mundiales. Ante el riesgo de aumentos en el precio internacional de maíz, México debe aumentar su producción en las zonas productoras. El déficit de maíz en Puebla es más agudo en la región central del estado (integrada por 88 municipios en los distritos de Cholula, Libres y Tecamachalco). En 2013 se sembraron en la región 327,000 ha de maíz, lo que representó 56.7 \% de la superficie total sembrada en el estado. Con un rendimiento de apenas $2.16 \mathrm{t} \mathrm{ha}^{-1}$ la región generó una producción de 705,000 t de maíz, lo que significó $74.8 \%$ del total estatal. En ese mismo año el consumo de maíz en la región fue de 830,000 t, $47.7 \%$ del total estatal; por tanto, la región tuvo un déficit de 125,000 $\mathrm{t}$.

El rendimiento observado en la región es una de las causas de la baja producción observada en la entidad, asociado a la tasa de adopción de semilla mejorada. Datos del SIAP (Com. Personal') indican que en 2013 la tasa promedio de adopción de semilla mejorada en la región central de Puebla fue de $37.46 \%$, pero la tasa de adopción es muy heterogénea en los 88 municipios que integran la región; por ejemplo, en 53 de los 88 municipios la tasa de utilización de

Servicio de Información Agroalimentaria y Pesquera (2012) Información proporcionada en la delegación SAGARPA del estado de Puebla. Julio de 2012 
semilla mejorada fue menor a $5 \%$ (SIAP, Com. Personal'). A nivel nacional la tasa promedio de adopción es un poco mayor: en el periodo 2008-2010 el consumo total nacional de semilla fue de 160,200 t, de las cuales $42.5 \%$ fue mejorada y el resto de criolla (García-Salazar y RamírezJaspeado, 2014).

La tasa de adopción de semilla mejorada depende de factores económicos, sociales y geográficos. Entre los factores más importantes que explican la tasa de adopción de semilla mejorada se pueden mencionar el tamaño de predio, el ingreso del productor, el precio de la semilla, y el precio de otros insumos como los fertilizantes y los plaguicidas (Bernard et al., 2010; García-Salazar y GuzmánSoria, 2015; Gecho y Punjabi, 2011; Kafle, 2010; Kalinda et al., 2014; Saín y Martínez, 1999; Serunkuuma, 2005;).

También se ha señalado que las principales causas por las cuales el agricultor usa semillas criollas son la adaptación de ésta a las condiciones climáticas, su rendimiento, el factor económico y la confianza y fe que los agricultores tienen en ella. Hellin et al. (2013) hacen énfasis en que existen varias razones, entre ellas las económicas, por las que los productores toman la decisión deliberada de sembrar maíces criollos. La causa que explica la siembra de variedades como maíz para pozole y maíz azul es el sobreprecio que se puede obtener si el productor accede a un mercado especializado, además de que no existe semilla mejorada para obtener los granos preferidos por el consumidor con calidades y colores diferentes. Por otro lado, las causas principales por las cuales unos productores usan semilla mejorada son: el probar o experimentar, el factor económico, el rendimiento y la resistencia al acame (Guillén-Pérez et al., 2002).

El mejoramiento genético y la innovación en semillas han sido cruciales en el aumento de la productividad de maíz (Donnet et al., 2012). En las zonas de temporal de México la demanda de semilla mejorada es heterogénea y el resultado de la adopción es incierto (Donnet et al., 2012). Las variedades mejoradas de maíz en México han estado disponibles por más de 50 años, y su difusión ha sido limitada (Bellon et al., 2006).

En el estado de Puebla se han implementado programas que han tenido como objetivo lograr la adopción de nuevas tecnologías por parte de los productores; por ejemplo, el Plan Puebla (Díaz et al., 1999). El aumento de la tasa de adopción de semilla mejorada es importante por el aumento que se puede lograr en la productividad, en la producción (García-Salazar y Ramírez-Jaspeado, 2013) y en el bienestar de los productores de maíz, lo cual implica que dicho aumento puede reducir la pobreza en los productores de maíz. La baja tasa de adopción puede dar una impresión errónea del verdadero impacto de germoplasma mejorado de maíz en el bienestar de los hogares; por tanto, es necesario medir las mejoras de la adopción de nuevas tecnologías.

La adopción de semilla mejorada implica un costo para el productor quien tiene que pagar por este insumo; sin embargo, la falta de conocimiento sobre los posibles beneficios que se tendrían en el rendimiento, en la producción, en el ingreso y en el bienestar es un factor determinante al momento de tomar la decisión de adoptar o no una tecnología. Bellon (1999) señala que el conocimiento de los pequeños agricultores en países en desarrollo es en muchas ocasiones utilizado para decidir sobre manejo agrícola, como adoptar o no una tecnología; sin embargo, este conocimiento tiene limitantes y lagunas que se pueden traducir en manejos y decisiones erróneas. En este sentido, es importante ampliar el conocimiento sobre los efectos positivos que tiene un aumento en la tasa de adopción.

Diversos estudios se han llevado a cabo sobre los efectos positivos que tiene la adopción de nuevas tecnologías en la reducción de la pobreza (Bellon et al., 2006; Binswanger y von Braun, 1991; Evenson y Gollin, 2003; Just y Zilberman, 1988); esta reducción puede darse a través de efectos directos e indirectos (De Janvry y Sadoulet, 2002). Investigaciones realizadas en países en desarrollo sobre los efectos que la adopción de semilla mejorada de maíz tiene sobre el bienestar de los hogares y el desarrollo rural indican que, en general, con la adopción se tiene un impacto positivo sobre productividad porque se reducen las importaciones, y los ingresos de los productores presentan beneficios tangibles por la venta de maíz, con lo que se reduce la pobreza extrema (Karanja et al., 2003; Morris et al., 1999). Los consumidores se ven beneficiados por los precios bajos que reciben como un efecto indirecto de la adopción (ISPC-SPIA, 2014). Para el caso de México, un estudio realizado por Becerril y Abdulai (2010) en Oaxaca y Chiapas indica que con la adopción de semilla mejorada de maíz se aumentó el gasto per capita promedio de los hogares, de 136 a 173 pesos.

Por la relevancia del tema, este trabajo tiene por objetivo medir los beneficios económicos de un aumento en la tasa de adopción de semilla mejorada de maíz sobre el bienestar de la sociedad, medido por el excedente económico (excedentes del productor y del consumidor) como resultado de cambios hipotéticos en el precio de la semilla mejorada, factor determinante en la tasa de adopción. El estudio trata de ampliar el conocimiento sobre los beneficios de aumentar la tasa de adopción a través de cambios en el pecio de la semilla mejorada. Se espera que aumentos en la tasa de utilización de semilla mejorada tengan un impacto positivo sobre el bienestar de los productores y 
consumidores de maíz en la región central del estado de Puebla.

\section{MATERIALES Y MÉTODOS}

El área de estudio fue la región central del estado de Puebla, integrada por 88 municipios ubicados en los distritos de Cholula, Libres y Tecamachalco, entre los paralelos $19^{\circ}$ $01^{\prime}$ y $19^{\circ} 07^{\prime} \mathrm{N}$, y los meridianos $98^{\circ} 16^{\prime}$ y $98^{\circ} 24^{\prime} \mathrm{O}$, con clima templado semi-seco. Para alcanzar el objetivo se usaron dos modelos: un modelo de equilibrio espacial (MEE) aplicado al mercado de maíz en Puebla, y un modelo logit agrupado (MLA) que determinó los factores que afectan la tasa de adopción de semilla mejorada en la región central de Puebla. La formulación del MEE se basó en la teoría económica del bienestar y los beneficios de la adopción se midieron a través del excedente económico (beneficio de la sociedad de producir y consumir maíz), o la suma de los excedentes al consumidor y al productor.

El bienestar se refiere al beneficio económico que tienen los vendedores y compradores cuando participan en el mercado del maíz, y se mide a través del excedente económico total (suma de los excedentes del productor y del consumidor). El excedente del productor es igual al ingreso que reciben los vendedores por los bienes que venden, menos sus costos de producción; se calcula descontando al ingreso el área bajo la curva de oferta. El excedente del consumidor es igual a la disposición de los compradores a pagar por un bien menos la cantidad que pagan realmente, o bien la diferencia entre la utilidad de los consumidores por consumir un bien menos el gasto pagado por adquirir el bien. Puede calcularse encontrando el área debajo de la curva de demanda y encima del precio. Ambos excedentes miden el beneficio que obtienen los vendedores y compradores que participan en un mercado (Krugman y Wells, 2013; Mankiw, 2002).

Se asume que existen seis regiones que producen y consumen maíz en el estado de Puebla, y que las regiones consumidoras se conectan a las zonas productoras del estado y fronteras de importación. Al considerar $d(d=1,2 \ldots D=6)$ regiones consumidoras de maíz, $s(s=1,2 \ldots S=6)$ regiones productoras y, $m(m=1=M=1)$ orígenes de importaciones, el modelo maximiza el bienestar de la sociedad (B):

$$
\begin{aligned}
\operatorname{MaxB}= & \sum_{d=1}^{D}\left[\lambda_{d} y_{d}+1 / 2 \omega_{d} y_{d}^{2}\right]-\sum_{s=1}^{s}\left[v_{s} x_{s}+1 / 2 \eta_{s} x_{s}^{2}\right]-\sum_{m=1}^{M}\left[p_{m} x_{m}\right] \\
& -\sum_{s=1}^{S} \sum_{d=1}^{D}\left[c t_{s d} x_{s d}\right]-\sum_{m=1}^{M} \sum_{d=1}^{D}\left[c t_{m d} x_{m d}\right]
\end{aligned}
$$

sujeto a:

$$
\sum_{s=1}^{s}\left[x_{s d}\right]+\sum_{m=1}^{M}\left[x_{m d}\right] \geq y_{d}
$$

$$
\begin{gathered}
\sum_{d=1}^{0}\left[x_{s d}\right] \leq x_{s} \\
\sum_{d=1}^{D}\left[x_{m d}\right] \leq x_{m} \\
y_{d^{\prime}} x_{s^{\prime}} x_{s d^{\prime}} x_{m d} \geq 0
\end{gathered}
$$

donde $y_{d}$ es el consumo de maíz en $d, \lambda_{d}$ es el intercepto de la función de demanda en $d, \omega_{d}$ es la pendiente de la función de demanda en $d, x_{s}$ es la producción en $s, v_{s}$ es el intercepto de la función de oferta en $s, \eta_{s}$ es la pendiente de la función de oferta en $s, p_{m}$ es el precio del maíz importado por $m, x_{m}$ son las importaciones por $m, c t_{s d}$ es el costo de transporte de $s$ a $d, x_{s d}$ es el envío de maíz de $s$ a $d, c t_{m d}$ es el costo de transporte de $m$ a $d, y x_{m d}$ es el envío de maíz de $m$ a d.

La Ecuación 1, que maximiza el bienestar (B), es igual al área bajo la curva de demanda, menos el área bajo la curva de oferta, el valor de las importaciones y los costos de transporte. Las Ecuaciones 2, 3 y 4 indican cómo se abastece el consumo de maíz de las regiones $d$, cómo se distribuye la producción de las zonas productoras $s, y$ cómo se distribuyen las importaciones de $m$. Se obtuvieron cuatro soluciones: la primera es la situación observada en 2013 y las otras tres (escenarios 1, 2 y 3) miden los beneficios de la adopción.

Para determinar los factores que afectan la tasa de adopción de semilla mejorada se usó un MLA para la región central de Puebla, en donde la tasa de adopción de semilla mejorada (o usar semilla criolla) se define como la probabilidad de usar semilla mejorada $\left(P_{\mathrm{i}}\right)$, o criolla en su caso $\left(1-P_{j}\right)$. El modelo se basó en García-Salazar y Guzmán-Soria (2015) y en Gujarati y Porter (2010), y consideró 88 municipios productores de maíz en Cholula, Libres y Tecamachalco. Al considerar $i(i=7,2, \ldots l=88)$ municipios productores de maíz, el MLA es el siguiente:

$$
\begin{aligned}
\sqrt{w_{i} L_{i}} & =\beta 1 \sqrt{w_{i}}+\beta 2 \sqrt{w_{i}} T_{A M}+\beta 3 \sqrt{w_{i}} \text { ING }_{i}+\beta 4 \sqrt{w_{i}} P S E_{i} \\
& +\beta 5 \sqrt{w_{i}} P F E_{i}+\beta 6 \sqrt{w_{i}} P O R G_{i}+\sqrt{w_{i}} u_{i}
\end{aligned}
$$


0

$$
\begin{aligned}
L_{i}^{*} & =\beta 7 \sqrt{w_{i}}+\beta 2 T A M_{i}^{*}+\beta 3 I N G_{i}^{*}+\beta 4 P S E_{i}^{*}+\beta S P F E_{i}^{*} \\
& +\beta 6 P O R G_{i}^{*}+v_{i}
\end{aligned}
$$

donde para $i, L_{i}$ es el logaritmo natural de razón de probabilidades de usar semilla mejorada y semilla criolla [Pi/ $(1-P i)]$, TAM $i$ es el tamaño de predio en hectáreas, ING es el ingreso en miles de $\$$ ha $^{-1}, P S E_{i}$ es el precio de la semilla mejorada en $\$ t^{-1}$ de maíz obtenido, $P F E_{i}$ es el precio del fertilizante en $\$ \mathrm{t}^{-1}, P O R G$, corresponde a unidades de producción integradas en organizaciones de productores, $u_{i}$ es el término de error, $\sqrt{W_{i}}=\sqrt{S T P_{i}\left(1-P_{i}\right)}$ es el ponderador, $L_{i}^{*}$ es $L_{i}$ transformada o ponderada, $T A M_{i}^{*}$ es el tamaño de predio ponderado, ING* es el ingreso ponderado, $P S E_{i}^{*}$ es el precio de la semilla ponderado, $P F E_{i}^{*}$ es el precio del fertilizante ponderado, $P O R G_{i}^{*}$ es el número de unidades integradas en organizaciones de productores ponderado y $v_{i}$ es el término de error ponderado.

La tasa de adopción de semilla mejorada se determinó a través de la probabilidad de usar semilla mejorada en la región $i\left(P_{j}\right)$, y ésta se calculó dividiendo la superficie sembrada con semilla mejorada $\left(S M_{i}\right)$ entre la superficie total de maíz (ST). La probabilidad de no usar semilla mejorada en la región $i$ es igual a 7-P. Una vez que el modelo se ha estimado se puede calcular la razón de probabilidades predichas (EC. 7), la probabilidad predicha de usar semilla mejorada (Ec. 8), y la probabilidad regional promedio de utilizar semilla mejorada (Ec. 9), esto es:

$$
\begin{aligned}
& \frac{\hat{P}_{i}}{1-\hat{P}_{i}}=e^{\hat{L}_{i}} \\
& \hat{P}_{i}=\frac{e^{\hat{L}_{i}}}{1+e^{\hat{L}_{i}}} \\
& \hat{P}_{s}=\sum_{i=1}^{1}\left[\hat{P}_{i} \times \delta_{i}\right]
\end{aligned}
$$

donde $\hat{P}_{s}$ es la probabilidad promedio de utilizar semilla mejorada en la región $s, \delta_{i}=\frac{S T_{i}}{S N T}$ es la participación de la región $i$ en la superficie total sembrada de maíz en $S$, $S T$. es la superficie sembrada de maíz en la región $i$, y SNT es la superficie sembrada total en la región central de Puebla.

Con base en las Ecs. 8 y 9 se obtuvo la probabilidad predicha de usar semilla mejorada en cada municipio y la probabilidad promedio de utilizar semilla mejorada (tasa de adopción) en la región central de Puebla. También se determinó cómo esta probabilidad se ve afectada cuando una de las variables exógenas experimenta un cambio.
Basados en García-Salazar y Ramírez-Jaspeado (2013), el efecto de la tasa de adopción de semilla mejorada sobre el rendimiento observado se calculó de la siguiente manera:

$$
r_{s}=r c_{s}\left(1-\hat{P}_{s}\right)+r m_{s} \hat{P}_{s}
$$

donde para la región $s, r_{s}$ es el rendimiento promedio observado de maíz en $t h^{-1}, r c_{s}$ es el rendimiento obtenido con semilla criolla en $t h a^{-1} y_{,} r m_{s}$ es el rendimiento obtenido con semilla mejorada en $\mathrm{tha}^{-1}$.

Mediante el uso del nuevo rendimiento $\left(r_{s}\right)$ y la superficie observada en el año base $\left(s_{s}\right)$, se obtuvo la nueva producción $\left(x_{s}=r_{s} \times s_{s}\right)$ para obtener una nueva solución del modelo (Ecs. 1 a 5). El escenario base del MEE consideró el rendimiento en 2013. Los escenarios 1, 2 y 3 consideraron el rendimiento estimado por cambios hipotéticos de 5 , 15 y $25 \%$, en el precio de la semilla mejorada de maíz. Es importante mencionar que no se asume que el precio de la semilla mejorada sea el único obstáculo para la adopción, sino que sólo se simulan escenarios donde se consideran cambios en el precio de la semilla mejorada, manteniendo constantes los demás factores que afectan la tasa de adopción. Las regiones usadas en el MEE se definieron considerando la clasificación de los distritos de riego reportados por el SIAP (2012a). Se consideraron las siguientes regiones: centro (integrada por 88 municipios), Huauchinango, Izúcar de Matamoros, Tehuacán, Teziutlán y Zacatlán. Se consideró un solo origen de las importaciones.

La producción de maíz se obtuvo del SIAP (2013). Basados en García-Salazar y Skaggs (2015) se realizó una estimación del consumo regional aparente y la información usada provino de García-Salazar y Ramírez-Jaspeado (2012), INEGI (2007), INEGI (2009), INEGI (2010) y SIAP (2012b). Se usaron matrices de distancias para calcular los costos de transporte con información de la Secretaria de Comunicaciones y Trasportes (SCT, 2012). La solución del MEE se obtuvo con el lenguaje de programación GAMS.

El MLA se estimó usando datos municipales de la región central de Puebla en 2011. Las fuentes de información respectivas fueron: SIAP (2011) para la superficie sembrada total de maíz, SIAP (Com. Personal') para la superficie sembrada de maíz que usa semilla mejorada, García-Salazar y Ramírez Jaspeado (2012) para la densidad de siembra por régimen hídrico e INEGI (2007) para el tamaño de predio de las unidades de producción de maíz y la organización del Censo Agropecuario.

Basados en García-Salazar y Guzmán-Soria (2015), se calculó el precio de la semilla mejorada en pesos por 
tonelada de maíz. La información sobre el precio de la semilla mejorada para estimar el precio por municipio se obtuvo del SNICS (2015), el rendimiento promedio municipal se obtuvo del SIAP (2011), y los precios de fertilizantes se obtuvieron del SNIIM (2015). La estimación del modelo se realizó usando el paquete SAS (SAS Institute, 1999). Se consideró que el nivel de rendimiento obtenido con semilla mejorada corresponde al rendimiento potencial, el cual se calculó con el mayor rendimiento municipal registrado en los municipios que integran la región central. Este último dato se obtuvo del SIAP (2012a).

\section{RESULTADOS Y DISCUSIÓN}

Los parámetros estadísticos a partir de la estimación del modelo logit en su forma ponderada se presentan en el Cuadro 1. El coeficiente de determinación $\left(R^{2}\right)$ del modelo fue de 0.65. La F calculada ( $F$ c) fue mayor a las $F$ de tablas (Ft) $(P<1 \%)$. El valor absoluto del valor de $t$ es igual o mayor a la unidad para los cinco parámetros, por lo que se puede decir que las variables usadas son significativas en el modelo.

Los coeficientes presentan el signo esperado de acuerdo con la teoría económica, pues los coeficientes del tamaño de predio, el ingreso y la organización presentan signo positivo, lo cual indica que un aumento en estas variables tendrá un efecto positivo sobre la probabilidad de usar semilla mejorada. Los parámetros de los precios de semilla mejorada y fertilizante con signo negativo, indican que una disminución en estas variables aumentará la probabilidad de usar semillas mejoradas (relación inversa) en la región central de Puebla. Los resultados obtenidos en el modelo son consistentes con el estudio realizado a nivel nacional por García-Salazar y Guzmán-Soria (2015), donde, con excepción de la variable organización, las demás variables que determinan la demanda de semilla mejorada tomadas en cuenta en este modelo presentan los mismos signos que a nivel nacional.

En el Cuadro 2 se presentan escenarios con cambios en el precio de la semilla mejorada. Estos cambios se realiza- ron en cada municipio perteneciente a la región central de Puebla, aunque aquí sólo se presenta la probabilidad promedio (TUM) de la región. La probabilidad promedio estimada para esta región fue 37.46 \%. Al emplear la Ecuación 10 se obtiene un rendimiento estimado de $2.2 \mathrm{t} \mathrm{ha}^{-1}$, que al multiplicarse por la superficie total $(326,801 \mathrm{ha})$ arroja una producción total igual a 705,000 t. La superficie total de maíz permaneció constante en cada escenario. En el modelo base el valor social neto (VSN) en Puebla equivale a 39,639 millones de pesos.

El precio de la semilla mejorada tiene una influencia negativa sobre la probabilidad de usar semilla mejorada. En el Escenario 1 el precio de la semilla mejorada de maíz disminuyó $5 \%$ en la región central de Puebla. Los efectos de tal cambio serían los siguientes: la tasa de utilización de usar semilla mejorada (TUM) en la región aumentaría 2.2 \% (al pasar de 37.4 a $38.3 \%$ ), el rendimiento crecería $0.8 \%$ (de 2.1 a $2.2 \mathrm{t} \mathrm{ha}^{-1}$ ), y la producción aumentaría de 705 a 711 mil toneladas. Finalmente, el bienestar de la sociedad aumentaría pues el VSN del estado aumentaría en $44.5 \mathrm{mi}-$ llones de pesos al crecer de 39,639 a 39,683 millones de pesos.

Al disminuir el precio de la semilla mejorada en $15 \%$ (Escenario 2), la tasa de utilización de usar semilla mejorada en la región central de Puebla aumentaría 6.6 \% respecto al escenario base. El rendimiento y la producción aumentarían 2.5 \% cada una. Por la mayor disminución en el precio de la semilla mejorada, el VSN del estado de Puebla aumentaría 133 millones de pesos. Cuando el precio de la semilla mejorada disminuye $25 \%$, la tasa de adopción aumentaría $11.1 \%$ y se alcanzaría $4.1 \%$ de aumento en la productividad; en este caso el VSN crecería 222 millones de pesos.

Los resultados de los modelos indican que la oferta en la región central de Puebla aumenta por una disminución en el precio de la semilla mejorada, lo que en consecuencia aumenta la tasa de adopción, el rendimiento y la producción, y elevan de manera significativa el bienestar de la sociedad consumidora de maíz. En México el precio de la

Cuadro 1. Resultados estadísticos del modelo logit para la región central de Puebla.

\begin{tabular}{lccccccc}
\hline Variable & Parámetro & Error estándar & Valor de $t$ & Prob $>|t|$ & $R^{2}$ & $F c$ & Prob $>F$ \\
\hline & 180.31879 & 40.48584 & 4.45 & $<.0001$ & 0.65 & 18.3 & $<.0001$ \\
TAM* & 0.44175 & 0.24136 & 1.83 & 0.0724 & & & \\
ING* & 0.12329 & 0.05527 & 2.23 & 0.0296 & & & \\
PSE* & -0.0011 & 0.000931 & -1.18 & 0.2424 & & & \\
PFE* & -0.03596 & 0.00799 & -4.5 & $<.0001$ & & & \\
ORG* & 0.01376 & 0.00779 & 1.77 & 0.0825 & & & \\
\hline
\end{tabular}

TAM = tamaño de predio; ING = ingreso; PSE = precio de la semilla; $P F E$ = precio del fertilizante; $O R G$ = número de unidades integradas en organizaciones de productores. 
Cuadro 2. Tasa de adopción, rendimiento, producción de maíz y bienestar en la región central de Puebla.

\begin{tabular}{|c|c|c|c|c|c|c|}
\hline Variable & TUM (\%) & TUC (\%) & Rendimiento (t ha-1) & Superficie (ha) & Producción (t) & VSN§ (miles de \$) \\
\hline Situación base & 37.46 & 0.6254 & 2.1569 & 326,801 & 704,873 & $39,639,000$ \\
\hline \multicolumn{7}{|c|}{ PSE disminuye en } \\
\hline $5 \%$ & 38.29 & 61.71 & 2.1746 & 326,801 & 710,664 & $39,683,491$ \\
\hline $15 \%$ & 39.95 & 60.05 & 2.2101 & 326,801 & 722,246 & $39,772,037$ \\
\hline $25 \%$ & 41.62 & 58.38 & 2.2457 & 326,801 & 733,898 & $39,860,832$ \\
\hline \multicolumn{7}{|c|}{ Cambio en \% respecto al modelo base } \\
\hline $5 \%$ & 2.2 & -1.3 & 0.8 & 0.0 & 0.8 & 0.1 \\
\hline $15 \%$ & 6.6 & -4.0 & 2.5 & 0.0 & 2.5 & 0.3 \\
\hline $25 \%$ & 11.1 & -6.7 & 4.1 & 0.0 & 4.1 & 0.6 \\
\hline
\end{tabular}

PSE = precio de la semilla mejorada en $\$ \mathrm{t}^{-1}$ de maíz obtenido; $\mathrm{TUM}=$ tasa de adopción de semilla mejorada; TUC = tasa de utilización de semilla criolla; VSN\& = valor social neto. TUM, TUC, rendimiento, superficie y producción corresponden a la región central de Puebla, y sólo el VSN§ corresponde al estado de Puebla.

semilla mejorada es alto. Al respecto se ha señalado que el monopolio en el comercio de las semillas propicia que los precios de venta de las semillas híbridas en México sean los más altos del mundo (Espinosa et al., 2010).

Por tanto, un menor precio en la semilla mejorada sólo se logrará si el mercado de dicha industria estuviera bajo competencia real. Un precio más bajo en la semilla mejorada podría proceder de un aumento en la cantidad ofrecida, de ahí la importancia de que las instituciones correspondientes eviten que esta industria se concentre en pocas empresas. Una forma de evitar la concentración de la producción en unas cuantas empresas sería la promoción de la producción de semilla mejorada por organizaciones de productores.

\section{CONCLUSIONES}

El aumento en la tasa de adopción de semilla mejorada de maíz en la región central de Puebla puede elevar de manera significativa el bienestar de los productores y consumidores. Una disminución en el precio de la semilla mejorada aumentaría el rendimiento, la producción y elevaría de manera significativa el bienestar de la sociedad consumidora de maíz en la región de estudio. Es necesario promover una estructura de mercado más competitiva, la cual induzca a la disminución en el precio de la semilla para elevar la tasa de adopción de semilla mejorada, el nivel de producción y el bienestar.

\section{BIBLIOGRAFÍA}

Becerril J. and A. Abdulai (2010) The impact of improved maize varieties on poverty in Mexico: a propensity score-matching approach. World Development 38:1024-1035.

Bellon M. R. (1999) La incorporación del conocimiento "local" del agri- cultor en los estudios de adopción de tecnología. In: La Adopción de Tecnologías. La Perspectiva del Agricultor y sus Implicaciones para la Elaboración de Políticas. G. Sain (ed.). Instituto Interamericano de Cooperación para la Agricultura (IICA). San José, Costa Rica. pp:43-52.

Bellon M. R., M. Adato, J. Becerril and D. Mindek (2006) Poor farmers' perceived benefits from different types of maize germplasm: the case of creolization in lowland tropical Mexico. World Development 34:113-129.

Bernard M., J. Hellin, R. Nyikal and J. Mburu (2010) Determinants for use of certified maize seed and the relative importance of transaction costs. Joint 3rd African Association of Agricultural Economists and 48th Agricultural Economists Association of South Africa Conference. Cape Town, South Africa, September 19-23. 26 p.

Binswanger H. P. and J. von Braun (1991) Technological change and commercialization in agriculture: The effect on the poor. The World Bank Research Observer 6:57-80.

De Janvry A. and E. Sadoulet (2002) World poverty and the role of agricultural technology: direct and indirect effects. The Journal of Development Studies 38:1-26.

Díaz C. H., L. Jiménez S., R. J. Laird y A. Turrent F. (1999) Plan Puebla, 25 Años de Experiencia: 1967-1992. Análisis de una Estrategia de Desarrollo de la Agricultura Tradicional. Colegio de Postgraduados. Montecillo, Estado de México. 174 p.

Donnet L., D. López, J. Arista, F. Carrión, V. Hernández y A. González (2012) El Potencial de Mercado de Semillas Mejoradas de Maíz en México. Documento de Trabajo 8. Programa de Socioeconomía, Centro Internacional de Mejoramiento de Maíz y Trigo, México. $21 \mathrm{p}$.

Espinosa C. A., M. Tadeo R. y A. Turrent F. (2010) Concentración de la oferta de semillas mejoradas de maíz. Periódico La Jornada, Sección La Jornada del Campo 13 de marzo de 2010. http://www.jornada. unam. mx /2010/03/13/oferta.html (Junio 2012).

Evenson R. E. and D. Gollin (2003) Crop genetic improvement in developing countries: overview and summary. In: Crop Variety Improvement and its Effect on Productivity: The Impact of International Agricultural Research. R. F. Everson and D. Gollin (eds.). CABI Publishing. Wallingford Oxon, UK. pp:7-38.

Flores-Cruz L. A., J. A. García-Salazar, J. S. Mora-Flores y F. Pérez-Soto (2014) Producción de maíz (Zea mays L.) en el estado de Puebla: un enfoque de equilibrio espacial para identificar las zonas productoras más competitivas. Agricultura Sociedad y Desarrollo 11:223-239.

García-Salazar J. A. y R. Ramírez-Jaspeado (2012) Demanda de Semilla Mejorada de Maíz en México: Identificación de Usos y Zonas de Producción con Mayor Potencial de Crecimiento. Centro Internacional de Mejoramiento de Maíz y Trigo (CIMMYT). Texcoco, 
Estado de México. 156 p.

García-Salazar J.A. y R. Ramírez-Jaspeado (2013) El tamaño de las unidades de producción de maíz: un desafío para elevar la tasa de utilización de semilla mejorada. Agrociencia 47:837-849.

García-Salazar J. A. y R. Ramírez-Jaspeado (2014) El mercado de la semilla mejorada de maíz (Zea mays L.) en México. Un análisis del saldo comercial por entidad federativa. Revista Fitotecnia Mexicana 37:69-77.

García-Salazar J. A. y E. Guzmán-Soria (2015) Factores que afectan la demanda de semilla mejorada de maíz en México. Revista Fitotecnia Mexicana 38:319-327.

García-Salazar J. A. and R. Skaggs (2015) Strategies for white and yellow maize cultivar improvement research and technology transfer in Mexico. Agronomy Journal 107:1425-1439.

Gecho Y. and N. K. Punjabi (2011) Determinants of adoption of improved maize technology in Damot Gale, Wolaita, Ethiopia. Rajasthan Journal of Extension Education 19:1-9.

Guillén-Pérez L. A., C. Sánchez-Quintanar, S. Mercado-Domenech y H. Navarro-Garza (2002) Análisis de atribución causal en el uso de semilla criolla y semilla mejorada de maíz. Agrociencia 36:377-387.

Gujarati D. N. y D. C. Porter (2010) Econometría. 5a ed. McGraw Hill. México, D. F. 921 p.

Hellin J., A. Keleman, D. López, L. Donnet y D. Flores (2013) La importancia de los nichos de mercado. Un estudio de caso del maíz azul y del maíz para pozole en México. Revista Fitotecnia Mexicana 36 (Especial 6):315-328.

INEGI, Instituto Nacional de Estadística, Geografía e Informática (2007) Censo agrícola, ganadero y forestal 2007. Aguascalientes, Ags. www.inegi.org.mx/est/contenidos/proyectos/Agro/ca2007/ Resultados_Agricola (Abril 2012)

INEGI, Instituto Nacional de Estadística, Geografía e Informática (2009) Censo económico. 2009. Aguascalientes, Ags. http://www.inegi. org.mx/est/contenidos/espanol/proyectos/censos/ce2009/ default.asp?s=est\&c=14220 (Mayo 2012).

INEGI, Instituto Nacional de Estadística, Geografía e Informática (2010) Censo de población y vivienda 2010. Aguascalientes, Ags. www. inegi.org.mx/est/contenidos/proyectos/ccpv/cpv2010/Default.aspx (Marzo 2012)

ISPC-SPIA, Independent Science and Partnership Council-Standing Panel on Impact Assessment (2014) Improved maize varieties and poverty in rural Ethiopia. Impact Brief No. 45. Consultative Group on International Agricultural Research (CGIAR). Rome, Italy, 4p.

Just R. E. and D. Zilberman (1988) The effects of agricultural development policies on income distribution and technological change in agriculture. Journal of Development Economics 28:193-216.

Kafle B. (2010) Determinants of adoption of improved maize varieties in developing countries: a review. International Research Journal of Applied and Basic Sciences 1:1-7.

Kalinda T., G. Tembo and E. Kuntashula (2014) Adoption of improved maize seed varieties in Southern Zambia. Asian Journal of Agricultural Sciences 6:33-39.

Karanja D. D., M. Renkow and E. W. Crawford (2003) Welfare effects of maize technologies in marginal and high potential regions of Kenya. Agricultural Economics 29:331-341.

Krugman P. y R. Wells (2013) Introducción a la Economía. Microeconomía. 2a ed. Ed. Reverté. Edición. Barcelona, España. 595 p.
Mankiw N. G. (2002) Principios de Economía. 2a ed. McGraw Hill. Madrid, España. 523 p.

Morris M. L., R. Tripp and A. A. Dankyi (1999) Adoption and Impacts of Improved Maize Production Technology: A case study of the Ghana grains development project. Economics Program Paper 99-01. CIMMYT. Mexico, D.F. 38 p.

Saín G. and J. Martínez (1999) Adoption and use of improved maize by small-scale farmers in Southeast Guatemala. Economic Paper 99-04. CIMMYT. México, D. F. 25 p.

SAS Institute (1999) SAS/ETS User's Guide, Version 8. Statistical Analysis System Institute Inc. Cary, NC, USA. 1546 p.

SCT, Secretaría de Comunicaciones y Transportes (2012) Rutas punto a punto. http://aplicaciones4.sct.gob.mx/sibuac_internet/Contro llerUl?action=cmdEscogeRuta. (Agosto 2012).

SIAP, Servicio de Información Agroalimentaria y Pesquera (2011) Información básica, agricultura, producción anual. Servicio de Información Agroalimentaria y pesquera. SAGARPA. México, D. F. www.siap.gob.mx/cierre-de-la-produccion-agricola-por-estado. (Agosto 2012).

SIAP, Servicio de Información Agroalimentaria y Pesquera (2012a) Información básica, agricultura, producción anual. Servicio de Información Agroalimentaria y Pesquera. SAGARPA. México, D. F. www. siap.gob.mx/cierre-de-la-produccion-agricola-por-estado (Marzo 2012)

SIAP, (Servicio de Información Agroalimentaria y Pesquera) (2012b) Información Básica, Agricultura, Balanza Nacional DisponibilidadConsumo. Servicio de Información Agroalimentaria y Pesquera. SAGARPA. México, D. F. www.siap.sagarpa.gob.mx/index.php? option=com_content\&view=article\&id $=12 \&$ Itemid=17 (Marzo 2012)

SIAP, Servicio de Información Agroalimentaria y Pesquera (2013) Información básica, agricultura, producción anual. Servicio de Información Agroalimentaria y pesquera. SAGARPA. México, D. F. www.siap.gob.mx/cierre-de-la-produccion-agricola-por-estado (Agosto 2015)

SIAP, Servicio de Información Agroalimentaria y Pesquera (2016) Información básica, agricultura, balanza nacional disponibilidad-consumo Servicio de Información Agroalimentaria y pesquera. SAGARPA. http://www.siap.gob.mx/balanza-disponibilidad-consumo/ (Mayo 2016)

SNICS, Servicio Nacional de Inspección y Certificación de Semillas (2015) Precio y existencias de semilla. SAGARPA. Tlanepantla, Edo. de México. http://snics.sagarpa.gob.mx/certificacion/Paginas / Precios_semilla_2011.aspx (Agosto 2015)

SNIIM, Servicio Nacional de Información e Integración de Mercados (2015) Precio de los insumos agrícolas. Secretaría de Economía. México, D. F. http://www.economia-sniim.gob.mx/SNIIM-AN/ estadisticas/e_anuariosinsu.asp. (Agosto 2015).

Sserunkuuma D. (2005) The adoption and impact of improved maize and land management technologies in Uganda. e-Journal of Agricultural and Development Economics 2:67-84.

USDA, Unites States Department of Agriculture (2016) USDA Agricultural Projections to 2025. Washinton, D. C. 93 p. www.usda.gov/ oce/commodity/projections/USDA_Agricultural_Projections_ to_2025.pdf (Mayo 2016). 